\title{
Efeito da música na pressão arterial: uma revisão sistemática
}

\author{
Effect of music on blood pressure: \\ a systematic review
}

\section{Jessica França Pereira' ${ }^{1}$ (1) Mayara Araujo de Souza ${ }^{2}$ (1) Fernanda Almeida de Assis ${ }^{3}$ (1) Priscilla Alfradique de Souza ${ }^{4}$ (1) Aline Affonso Luna 5 (1) Natália Chantal Magalhães da Silva ${ }^{6}$ (1)}

\footnotetext{
1-5Universidade Federal do Estado do Rio de Janeiro (Rio de Janeiro). Rio de Janeiro, Brasil. jessica.france.p@gmail.com, mayara.araujods@gmail.com, fernandaalmeida142@gmail.com, priscilla.souza@unirio.br, aline.luna@unirio.br ${ }^{6}$ Autora para correspondência. Universidade Federal do Estado do Rio de Janeiro (Rio de Janeiro). Rio de Janeiro, Brasil. natalia.c.silva@unirio.br
}

\begin{abstract}
RESUMO | OBJETIVO: Analisar o efeito da música na pressão arterial. MÉTODO: Revisão sistemática, realizada a partir da estratégia PRISMA, que teve como questão norteadora "Em relação à pessoas que apresentam ou não o diagnóstico de hipertensão arterial sistêmica, qual o efeito da música, comparado à intervenção padrão, sobre a pressão arterial?". A seleção e extração de informações foram realizadas no período de outubro a dezembro de 2019, nas bases de dados PubMed Central, Cochrane Central, Science Direct e Scientific Electronic Library Online (SciELO). Foi utilizada como estratégia de busca: "Music" and "Hypertension" or "Arterial Pressure" and "Randomized Controlled Trial". RESULTADOS: Após a análise seletiva e crítica dos achados, 14 ensaios compuseram esta revisão. Desses, 10 indicaram efeito da intervenção na pressão arterial, sendo observada diminuição significativa na pressão arterial sistólica. Identificou-se, ainda, que a maioria dos estudos que utilizam a música como terapia de controle da pressão arterial foi em pacientes adultos, em tratamento do câncer ou procedimentos cirúrgicos. CONCLUSÃO: Os estudos analisados evidenciaram que a música reduziu os níveis pressóricos e pode ser considerada um recurso para o controle da pressão arterial. Entretanto, são necessários estudos que verifiquem os efeitos da intervenção a longo prazo e que sigam protocolos de avaliação da pressão arterial.
\end{abstract}

DESCRITORES: Terapias Complementares. Música. Pressão arterial.

\begin{abstract}
OBJECTIVE: To analyze the effect of music on blood pressure. METHOD: Systematic review, carried out based on The PRISMA, whose guiding question was "In relation to people who present or not the diagnosis of systemic arterial hypertension, what is the effect of music, compared to standard intervention, on blood pressure?". The selection and extraction of information was carried out from October to December 2019, in the PubMed Central, Cochrane Central, Science Direct and Scientific Electronic Library Online (SciELO) databases. The search strategy used was: "Music" and "Hypertension" or "Arterial Pressure" and "Randomized Controlled Trial". RESULTS: After the selective and critical analysis of the findings, 14 trials comprised this review. Of these, 10 indicated the effect of the intervention on blood pressure, with a significant decrease in systolic blood pressure. It was also identified that the majority of studies that use music as a blood pressure control therapy were in adult patients, undergoing cancer treatment or surgical procedures. CONCLUSION: The studies analyzed showed that music reduced blood pressure levels and can be considered a resource for controlling blood pressure. However, studies are needed to verify the effects of long-term intervention that follow blood pressure assessment protocols.
\end{abstract}

DESCRIPTORS: Complementary Therapies. Music. Arterial pressure. 


\section{Introdução}

No Brasil, as doenças crônicas não transmissíveis (DCNT) acometem cerca de $45 \%$ da população adulta e causam, aproximadamente, 41 milhões de mortes no mundo ${ }^{1}$. Elas são consequências de uma combinação de fatores genéticos, fisiológicos, ambientais e comportamentais e tendem a ser de longa duração?

Segundo a Organização Mundial da Saúde, as doenças cardiovasculares são as principais responsáveis pelas mortes por DCNT, sendo a pressão arterial elevada seu principal fator de risco ${ }^{1}$. A pressão arterial pode ser definida como a "força exercida pelo sangue contra qualquer unidade de área da parede vascular"3. Ela é determinada pelo produto da resistência vascular periférica e do débito cardíaco e regulada por ações dos sistemas neural, renal, cardiovascular e endócrino. Assim, a instabilidade de uma dessas constantes ou de um dos sistemas reguladores pode levar ao aumento da pressão arterial .

A elevação constante dos níveis pressóricos - igual ou acima de 140 (pressão arterial sistólica - PAS) e/ ou 90 (pressão arterial diastólica - PAD) mmHg - configura-se uma condição clínica caracterizada por hipertensão arterial sistêmica - HAS. Tal condição de saúde é associada a distúrbios metabólicos e alterações funcionais ou estruturais de órgãos-alvo, sendo agravada por fatores de risco. Entre estes fatores, destaca-se idade, sexo, etnia, excesso de peso, ingestão de sal, ingestão de álcool, sedentarismo, fatores considerados socioeconômicos e genéticos ${ }^{5}$.

No Brasil, a doença está presente em $24,7 \%$ da população, sendo que um a cada quatro adultos possui o diagnóstico ${ }^{6-7}$, sua abordagem terapêutica inclui medidas medicamentosas e não medicamentosas com objetivo de reduzir e controlar a pressão arterial. Entretanto, destaca-se que a escolha do tratamento precisa levar em consideração fatores intrínsecos (hereditariedade, etnia, sexo, idade) e extrínsecos (hábitos de vida, alimentação, sedentarismo, tabagismo) que podem desencadear a alteração sistêmica. A conduta deve ser individualizada e pautada no princípio da integralização ${ }^{5,8}$.
Nesse contexto, a utilização das Práticas Integrativas e Complementares (PICs) surgem como uma possibilidade de complementação ao tratamento convencional. Também conhecidas como terapias alternativas, podem ser definidas como um conjunto de práticas de saúde fundamentadas na humanização e integralização da assistência que visam a prevenção, promoção, manutenção e recuperação da saúde? .

Desde a década de 70, com a criação do Programa de Medicina Tradicional, há um incentivo à criação e à implementação de políticas públicas que estimulem o uso das PICs. No Brasil, o reconhecimento e a institucionalização das práticas iniciaram na década de 80, principalmente, após a criação do SUS. Todavia, a normatização ocorreu somente em 2006 por meio da Portaria $n^{\circ}$ 971, que aprovou a Política Nacional de Práticas Integrativas e Complementares ${ }^{10}$. Atualmente, o Ministério da Saúde reconhece a existência de 29 PICs, dentre elas, a musicoterapia ou a terapia com música ${ }^{11}$.

A terapia utiliza a música e seus elementos - som, ritmo, melodia e harmonia - como intervenção? . Ela é considerada uma tecnologia de baixo custo e sua utilização vem se tornando cada vez mais comum nos serviços de saúde, sendo utilizada como recurso terapêutico por diversos profissionais, inclusive enfermeiros ${ }^{12-13}$.

Existem três possibilidades de utilização da música como uma intervenção: receptiva (o ouvinte escuta a música produzida ou músicas previamente gravadas), ativa (o ouvinte utiliza instrumentos musicais ou a voz para produzir sons musicais) e interativa (a experiência musical é realizada em grupo) $)^{14}$.

Com a finalidade de comprovar seus efeitos, pesquisas na área vêm sendo incentivadas. Alguns resultados evidenciam os benefícios da música sobre o controle de parâmetros emocionais, sociais e biológicos ${ }^{12-14}$. Contudo, existe uma lacuna na literatura sobre estudos que investigam o uso da música no controle da pressão arterial. 
Nesse contexto, e dado a necessidade de utilização de recursos não usuais como coadjuvantes aos tratamentos convencionais, este estudo teve como objetivo analisar o efeito da música no controle da pressão arterial.

\section{Método}

Trata-se de uma revisão sistemática que partiu da seguinte questão norteadora elaborada por meio da estratégia PICO: “Em relação à pessoas que apresentam ou não o diagnóstico de hipertensão arterial sistêmica (population), qual o efeito da música (intervention), comparado à intervenção padrão (control), sobre a pressão arterial (outcomes)?".

A busca foi realizada no período de outubro a dezembro de 2019, nas bases de dados PubMed Central, Cochrane Central, Science Direct e Scientific Electronic Library Online (SciELO). Com o objetivo de minimizar o viés, o processo de seleção dos artigos e extração das informações foi realizado por dois pesquisadores e, na presença de desacordos, um terceiro pesquisador foi consultado.

Foi utilizada como estratégia de busca os seguintes descritores presentes no Medical Subject Headings (MeSH): "Music" and "Hypertension" or "Arterial pressure" and "Randomized Controlled trial".

Como limites (critérios de inclusão), foram considerados: ensaios clínicos randomizados publicados nos últimos seis anos (uma vez que foi realizada uma revisão da temática em 2014 ${ }^{13}$ ), disponíveis em texto completo e em língua portuguesa, inglesa e espanhola.
Foram excluídos aqueles que não atendiam à temática abordada, não respondiam à questão norteadora e que estavam em duplicidade.

Os estudos encontrados foram analisados em duas etapas. Na primeira etapa, foi realizada a análise seletiva com a leitura dos títulos e resumos, havendo exclusão daqueles que não atendiam a temática abordada. Em seguida, na segunda etapa, ocorreu a análise crítica, que consistiu na leitura na íntegra dos estudos selecionados na etapa anterior, sendo excluídos aqueles que não respondiam à questão norteadora e estavam em duplicidade.

Os dados presentes nos ensaios foram analisados a partir de um formulário eletrônico no Excel, em um modelo criado especificamente para este estudo com itens relacionados a elementos de identificação do artigo (autores, título, ano e país de publicação) e características gerais (objetivos, métodos, participantes, procedimentos de coleta de dados, protocolo de avaliação da pressão arterial, protocolo de aplicação da música, resultados e conclusão).

Ademais, com a finalidade de melhorar a qualidade metodológica desta revisão, foi seguida a estratégia Preferred Reporting Items for Systematic Reviews and Meta-Analyses - The PRISMA Statement ${ }^{15}$ e aplicada, aos ensaios, a escala de qualidade metodológica proposta por Jadad ${ }^{16}$.

\section{Resultados}

Após a aplicação da estratégia de busca e observação dos critérios de elegibilidade, 14 artigos compuseram esta revisão sistemática (Figura 1). 


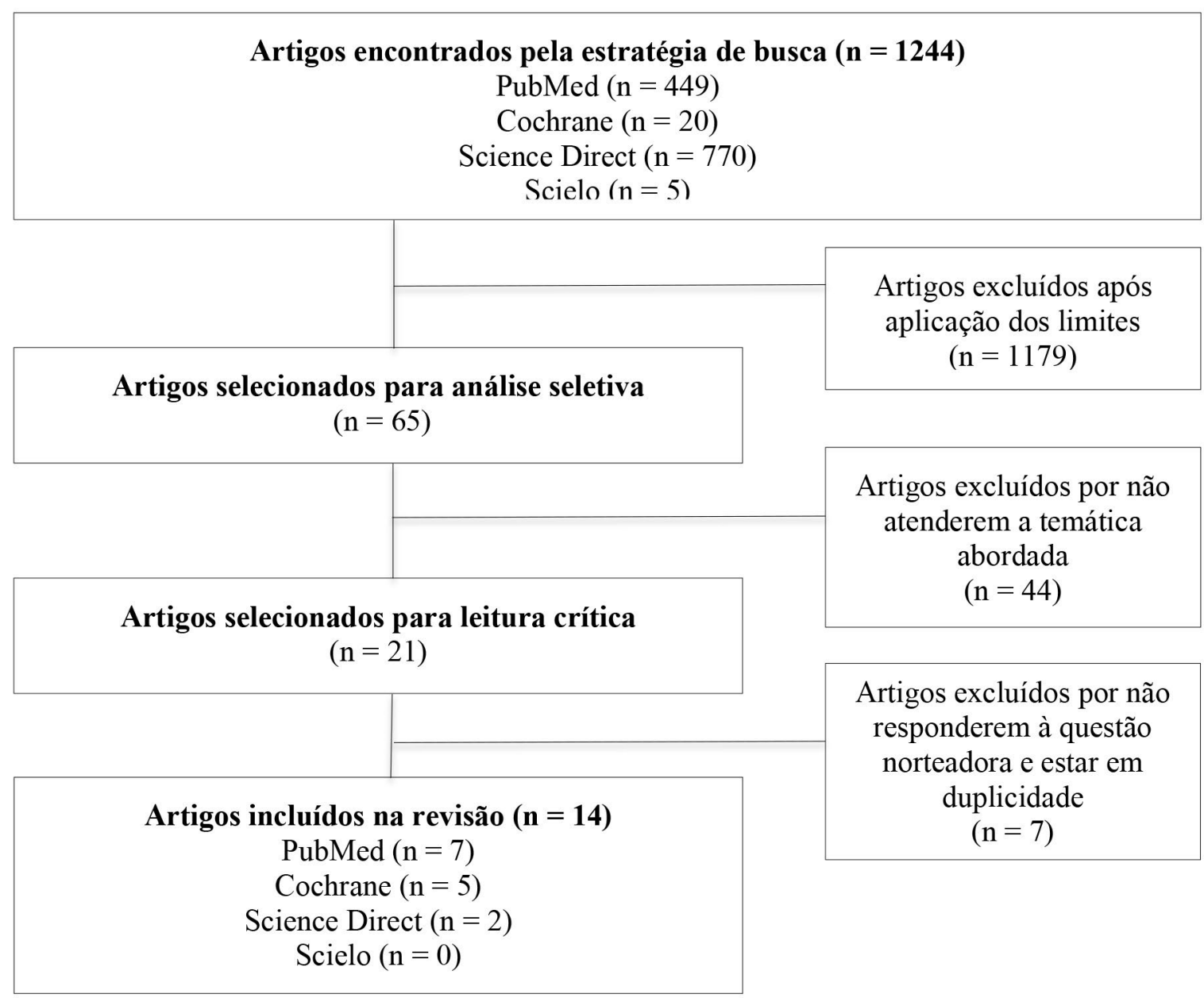


A pressão arterial foi avaliada de formas diferentes nos estudos. Dos artigos selecionados, um utilizou esfigmomanômetro e estetoscópio ${ }^{17}$, um, monitor de pressão digital 1819 e três, monitor multiparamétri$\mathrm{CO}^{20-22}$. Nos demais estudos não foram informados o recurso utilizado e a metodologia utilizada para mensuração. Os intervalos de avaliação variaram de uma pesquisa para outra, mas todas realizaram a mensuração da pressão arterial em pelo menos dois momentos distintos, antes e após a intervenção.

O tempo de reprodução da música foi relatado em 10 dos 14 estudos que compuseram esta revisão, tendo como variação o intervalo de 10 a 60 minutos $12,17,19,20$, $22,23-27$.

Em relação ao número de sessões ofertadas, em 12 ensaios houve fornecimento de apenas uma sessão 12,17,18,20,22,23-29, em um, duas sessões ${ }^{21}$, e em outro, a música foi fornecida durante 60 dias consecutivos, duas vezes ao dia ${ }^{19}$.

Em $85 \%$ dos estudos, a música foi ofertada de forma receptiva (individualizada) por meio de fones de ouvido; nos demais, a oferta ocorreu de modo interativo (em grupo). Foi evidenciada variação de estilo musical, contudo, a música clássica se fez presente em cinco estudos $12,17,20,27,29$.
Em relação aos efeitos da música, 10 ensaios exibi-

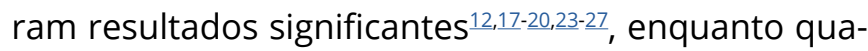
tro afirmaram que a música não apresentou efeito sobre a pressão arterial 121,22,28,29. Nos estudos em que houve efeito da terapia, foi observada diminuição significativa apenas na PAS.

Dentre os artigos que apresentaram efeito da música na diminuição na variável de interesse, somente quatro estudos não envolviam procedimentos cirúrgicos $12,17,19,23$. Em tais pesquisas, a população alvo foi composta por pessoas saudáveis em acompanhamento ambulatorial12, que realizavam hemodiálise ${ }^{17}$, que apresentaram infarto agudo do miocárdio $\frac{19}{} \mathrm{e}$ crianças em ventilação mecânica ${ }^{23}$.

As implicações da música na área da saúde foram descritas em cinco estudos $17,18,26,27,29$. Nestes, houve um consenso de que a intervenção é uma tecnologia leve, de baixo custo e de fácil aplicação.

O Quadro 1 apresenta as informações mais relevantes dos ensaios clínicos randomizados que compuseram a revisão sistemática. 
Quadro 1. Ensaios clínicos randomizados que compuseram a revisão sistemática. Rio de Janeiro, RJ, Brasil, 2020 (continua)

\begin{tabular}{|c|c|c|c|c|}
\hline Autores & Objetivos & $\begin{array}{l}\text { Protocolo de } \\
\text { aplicação da } \\
\text { música }\end{array}$ & Efeito na pressão arterial & $\begin{array}{c}\text { Escore de } \\
\text { Jadad }^{16}\end{array}$ \\
\hline $\begin{array}{l}\text { Melo GAA, } \\
\text { Rodrigues AB, } \\
\text { Grangeiro ASM, } \\
\text { Oliveira PP, } \\
\text { Caetano JA }\end{array}$ & $\begin{array}{l}\text { Avaliar o efeito de uma } \\
\text { intervenção musical sobre a } \\
\text { ansiedade e parâmetros } \\
\text { vitais em doentes renais } \\
\text { crônicos em comparação ao } \\
\text { cuidado convencional de } \\
\text { clínicas de hemodiálise. }\end{array}$ & $\begin{array}{l}\text { Uma sessão de } \\
30 \text { minutos de } \\
\text { modo } \\
\text { interativo. }\end{array}$ & $\begin{array}{l}\text { Houve diminuição da PAS } \\
\text { significativamente maior no } \\
\text { GE e não houve diferença } \\
\text { entre o GE e GC para PAD. }\end{array}$ & V \\
\hline $\begin{array}{l}\text { Lin, DC, } \\
\text { Marinova M, } \\
\text { Rubino G, Gola } \\
\text { E, Brocca A, } \\
\text { Pantano G }\end{array}$ & $\begin{array}{l}\text { Estudar alguns possíveis } \\
\text { sinais através dos quais a } \\
\text { resposta relaxante } \\
\text { (provocada pela meditação e } \\
\text { escuta musical) influencia a } \\
\text { expressão gênica e muitos } \\
\text { parâmetros cardiovasculares } \\
\text { clínicos, morfológicos, } \\
\text { estruturais e funcionais. }\end{array}$ & $\begin{array}{l}120 \text { sessões de } \\
20 \text { minutos, } \\
\text { (duas vezes ao } \\
\text { dia, por } 60 \text { dias) } \\
\text { de modo } \\
\text { receptivo. }\end{array}$ & A PAS foi reduzida no GE. & IV \\
\hline $\begin{array}{l}\text { Firmeza MA, } \\
\text { Rodrigues AB, } \\
\text { Melo GAA, } \\
\text { Aguiar MIF, } \\
\text { Cunha GH, } \\
\text { Oliveira PP, } \\
\text { Grangeir ASM }\end{array}$ & $\begin{array}{l}\text { Avaliar a eficácia de uma } \\
\text { intervenção musical na } \\
\text { redução da ansiedade e } \\
\text { parâmetros vitais em } \\
\text { pessoas que sofrem de } \\
\text { câncer de cabeça e pescoço. }\end{array}$ & $\begin{array}{l}\text { Uma sessão de } \\
30 \text { minutos de } \\
\text { modo } \\
\text { receptivo. }\end{array}$ & $\begin{array}{l}\text { 95,0\% do GE apresentou } \\
\text { redução da PAS, 55,0\% } \\
\text { exibiram redução da PAD, } \\
\text { representando uma } \\
\text { diminuição média de 10,95 } \\
\text { mmHg na PAS e de 3,85 } \\
\text { mmHg na PAD. }\end{array}$ & III \\
\hline $\begin{array}{l}\text { Lee, WP, Wu PY, } \\
\text { Lee MY, Ho LH, } \\
\text { Shih WM }\end{array}$ & $\begin{array}{l}\text { Explorar os efeitos da } \\
\text { audição de música nos } \\
\text { níveis de ansiedade e } \\
\text { respostas fisiológicas de } \\
\text { pacientes cirúrgicos sob } \\
\text { raquianestesia. }\end{array}$ & $\begin{array}{l}\text { Uma sessão de } \\
30 \text { minutos de } \\
\text { modo } \\
\text { receptivo. }\end{array}$ & $\begin{array}{l}\text { O GE apresentou } \\
\text { diminuição de PAS e PAD } \\
\text { após a intervenção, o que } \\
\text { não foi observado no GC, } \\
\text { sendo essa diferença } \\
\text { intergrupos } \\
\text { estatisticamente } \\
\text { significativa. }\end{array}$ & III \\
\hline $\begin{array}{l}\text { Wu PY, Huang } \\
\text { ML, Lee WP, } \\
\text { Wang C, Shih } \\
\text { WM }\end{array}$ & $\begin{array}{l}\text { Explorar os efeitos da escuta } \\
\text { musical no nível de } \\
\text { ansiedade e respostas } \\
\text { fisiológicas da craniotomia } \\
\text { acordada. }\end{array}$ & $\begin{array}{l}\text { Uma sessão de } \\
30 \text { minutos de } \\
\text { modo } \\
\text { receptivo. }\end{array}$ & $\begin{array}{l}\text { Após a intervenção musical, } \\
\text { o teste indicou que a PAS e } \\
\text { PAD entre os dois grupos } \\
\text { apresentaram diferença } \\
\text { significante, com maiores } \\
\text { médias no GC e menores } \\
\text { médias no GE. }\end{array}$ & III \\
\hline $\begin{array}{l}\text { Ripley L, } \\
\text { Christopoulos } \\
\text { G, Michael TT, } \\
\text { Alomar M, } \\
\text { Rangan BV, } \\
\text { Roesle M et al }\end{array}$ & $\begin{array}{l}\text { Determinar o impacto da } \\
\text { intervenção musical na } \\
\text { função endotelial, } \\
\text { hemodinâmica e ansiedade } \\
\text { do paciente antes, durante e } \\
\text { após o cateterismo cardíaco. }\end{array}$ & $\begin{array}{l}\text { Duas sessões } \\
\text { (uma antes e } \\
\text { outra depois do } \\
\text { cateterismo) de } \\
\text { modo } \\
\text { receptivo. }\end{array}$ & $\begin{array}{l}\text { O impacto da música nos } \\
\text { parâmetros } \\
\text { hemodinâmicos foi } \\
\text { semelhante entre os dois } \\
\text { grupos, não havendo } \\
\text { diferença estatisticamente } \\
\text { significante. }\end{array}$ & III \\
\hline $\begin{array}{l}\text { Choi S, Park SG, } \\
\text { Bellan L, Lee } \\
\text { HH, Chung SK }\end{array}$ & $\begin{array}{l}\text { Determinar o efeito da } \\
\text { música sobre a dor } \\
\text { experimentada por } \\
\text { pacientes coreanos } \\
\text { submetidos a cirurgia } \\
\text { sequencial bilateral de } \\
\text { catarata. }\end{array}$ & $\begin{array}{l}\text { Uma sessão } \\
\text { durante a } \\
\text { cirurgia, de } \\
\text { modo } \\
\text { receptivo. }\end{array}$ & $\begin{array}{l}\text { Comparações para o GE e } \\
\text { GC não atingiram } \\
\text { significância estatística. A } \\
\text { intervenção com música foi } \\
\text { mais eficaz para pacientes } \\
\text { com pressão arterial acima } \\
\text { dos parâmetros normais. }\end{array}$ & III \\
\hline
\end{tabular}


Quadro 1. Ensaios clínicos randomizados que compuseram a revisão sistemática. Rio de Janeiro, RJ, Brasil, 2020 (conclusão)

\begin{tabular}{|c|c|c|c|c|}
\hline Autores & Objetivos & $\begin{array}{l}\text { Protocolo de } \\
\text { aplicação da } \\
\text { música }\end{array}$ & Efeito na pressão arterial & $\begin{array}{c}\text { Escore de } \\
\text { Jadad }^{16}\end{array}$ \\
\hline $\begin{array}{l}\text { Kahloul M, } \\
\text { Mhamdi S, } \\
\text { Nakhi MS, } \\
\text { Steyhi NA, } \\
\text { Azzaza M, } \\
\text { Chaouch A, } \\
\text { Naija W }\end{array}$ & $\begin{array}{l}\text { Avaliar os efeitos da } \\
\text { musicoterapia na satisfação, } \\
\text { estresse, dor e } \\
\text { conscientização de pacientes } \\
\text { em perioperatório. }\end{array}$ & $\begin{array}{l}\text { Uma sessão } \\
\text { imediatamente } \\
\text { após a indução } \\
\text { da anestesia, } \\
\text { de modo } \\
\text { receptivo. }\end{array}$ & $\begin{array}{l}\text { A comparação dos dois } \\
\text { grupos em relação ao perfil } \\
\text { hemodinâmico encontrou } \\
\text { maior estabilidade no GE } \\
\text { somente para PAS, } \\
\text { particularmente em } 10 \text { e } 30 \\
\text { minutos após a indução } \\
\text { anestésica. }\end{array}$ & IV \\
\hline $\begin{array}{l}\text { Trappe HJ, Voit } \\
\text { G }\end{array}$ & $\begin{array}{l}\text { Testar o efeito de diferentes } \\
\text { estilos musicais nos níveis } \\
\text { séricos de cortisol, pressão } \\
\text { arterial e freqüência } \\
\text { cardíaca é atualmente } \\
\text { desconhecido. }\end{array}$ & $\begin{array}{l}\text { Uma sessão de } \\
25 \text { minutos de } \\
\text { modo } \\
\text { receptivo. }\end{array}$ & $\begin{array}{l}\text { Houve diminuição } \\
\text { significativa na PAS e PAD } \\
\text { do GE. }\end{array}$ & III \\
\hline $\begin{array}{l}\text { Wieatwongwan } \\
\text { a D, } \\
\text { Vichitvejpaisal } \\
\text { P, Thaikruea L, } \\
\text { Klaphajone J, } \\
\text { Tantong A, } \\
\text { Wiwatwongwan } \\
\text { a A }\end{array}$ & $\begin{array}{l}\text { Investigar os efeitos } \\
\text { ansiolíticos da música em } \\
\text { pacientes submetidos à } \\
\text { cirurgia de catarata sob } \\
\text { anestesia local. }\end{array}$ & $\begin{array}{l}\text { Uma sessão de } \\
10 \text { minutos de } \\
\text { modo } \\
\text { receptivo. }\end{array}$ & $\begin{array}{l}\text { A PAS foi significativamente } \\
\text { menor no GE do que no GC, } \\
\text { havendo aumento da PAS } \\
\text { durante a cirurgia. }\end{array}$ & v \\
\hline $\begin{array}{l}\text { Liu MH, Zhu LH, } \\
\text { Peng JX, Zhang } \\
\text { XP, Xiao ZH, Liu } \\
\text { QJ, Latour JM }\end{array}$ & $\begin{array}{l}\text { Determinar a viabilidade de } \\
\text { uma intervenção musical } \\
\text { personalizada com crianças } \\
\text { sob ventilação mecânica na } \\
\text { UTI pediátrica. }\end{array}$ & $\begin{array}{l}\text { Uma sessão de } \\
60 \text { minutos de } \\
\text { modo } \\
\text { interativo. }\end{array}$ & $\begin{array}{l}\text { No primeiro momento (5 } \\
\text { minutos antes da } \\
\text { intervenção), não houve } \\
\text { diferenças na PA entre os } \\
\text { grupos. Já no momento } \\
\text { após a intervenção (5 } \\
\text { minutos após), houve } \\
\text { melhora, significativa, da } \\
\text { PAS do GE. }\end{array}$ & III \\
\hline $\begin{array}{l}\text { Ko SY, Leung } \\
\text { DY, Wong EM }\end{array}$ & $\begin{array}{l}\text { Examinar os efeitos da } \\
\text { música de fácil audição nos } \\
\text { requisitos de satisfação, } \\
\text { ansiedade, dor, } \\
\text { medicamentos sedativos, } \\
\text { analgésicos e parâmetros } \\
\text { fisiológicos em pacientes } \\
\text { adultos submetidos à } \\
\text { colonoscopia. }\end{array}$ & $\begin{array}{l}\text { Uma sessão de } \\
20 \text { minutos } \\
\text { antes e durante } \\
\text { o } \\
\text { procedimento, } \\
\text { com } 15 \text { músicas } \\
\text { tocadas, de } \\
\text { modo } \\
\text { receptivo. }\end{array}$ & $\begin{array}{l}\text { Não houve diferenças } \\
\text { estatísticas entre o GE e GC } \\
\text { em termos de PAS e PAD. }\end{array}$ & $\mathrm{v}$ \\
\hline $\begin{array}{l}\text { Ortega A, } \\
\text { Gauna F, } \\
\text { Munoz D, } \\
\text { Oberreuter G, } \\
\text { Breinbauer HÁ, } \\
\text { Carrasco L }\end{array}$ & $\begin{array}{l}\text { Avaliar se ouvir música } \\
\text { através de fones de ouvido } \\
\text { binaurais contribui para a } \\
\text { percepção de dor e } \\
\text { ansiedade em pacientes } \\
\text { submetidos a reduções } \\
\text { fechadas de fraturas ósseas } \\
\text { nasais. }\end{array}$ & $\begin{array}{l}\text { Uma sessão de } \\
20 \text { minutos } \\
\text { durante todo o } \\
\text { procedimento e } \\
\text { no pós- } \\
\text { operatório, de } \\
\text { modo } \\
\text { receptivo. }\end{array}$ & $\begin{array}{l}\text { No GC, a PAS aumentou } \\
\text { quando a anestesia local foi } \\
\text { aplicada. Por outro lado, o } \\
\text { grupo exposto à música } \\
\text { manteve a PAS estável } \\
\text { durante toda a experiência. }\end{array}$ & V \\
\hline $\begin{array}{l}\text { Kavakli AS, } \\
\text { Ozturk NK, } \\
\text { Adas HY, } \\
\text { Kudsioglu ST }\end{array}$ & $\begin{array}{l}\text { Testar a hipótese de que } \\
\text { ouvir música durante a } \\
\text { endarterectomia de carótida } \\
\text { (CEA) sob anestesia regional } \\
\text { diminui a ansiedade e a dor } \\
\text { do paciente. }\end{array}$ & $\begin{array}{l}\text { Uma sessão } \\
\text { durante todo o } \\
\text { procedimento } \\
\text { (interrompida } \\
\text { antes de cada } \\
\text { avaliação e } \\
\text { retomada logo } \\
\text { após), de modo } \\
\text { receptivo. }\end{array}$ & $\begin{array}{l}\text { A PAS e a PAD não } \\
\text { apresentaram diferença } \\
\text { significativa nos GE e GC } \\
\text { nos momentos do período } \\
\text { intraoperatório. }\end{array}$ & IV \\
\hline
\end{tabular}

PAS: Pressão arterial sistólica; PAD: Pressão arterial diastólica; GE: Grupo Experimental; GC: Grupo Controle. 


\section{Discussão}

O número reduzido de ensaios que testaram o efeito da música na pressão arterial corrobora com a literatura científica e, ao mesmo tempo, revela ser incipiente o uso certificado da música como prática integrativa e complementar na área da saúde ${ }^{13,14,31,32}$.

Nesta revisão, apenas seis estudos relataram o equipamento utilizado para mensurar a pressão arterial17-22, sendo que em três foi utilizado o monitor multiparamétrico ${ }^{20-22}$. Destaca-se que tais informações se concentraram na exposição do equipamento utilizado, sem detalhar a metodologia para mensuração da variável.

Segundo a Sociedade Brasileira de Cardiologia ${ }^{5}$, a pressão arterial pode ser mensurada de forma manual ou eletrônica. Manualmente é a forma mais comumente realizada, sendo utilizado o esfigmomanômetro e o estetoscópio. No formato eletrônico, usualmente empregado durante procedimentos, cirurgias ou em pacientes que precisam ter seus sinais fisiológicos monitorados devido às condições específicas, podem ser utilizados equipamentos de mensuração intermitente, como: esfigmomanômetros portáteis e automáticos e equipamentos para monitorização ambulatorial da pressão arterial MAPA e monitorização residencial da pressão arterial - MRPA; além de equipamentos de mensuração contínua, como os monitores multiparamétricos.

Os ensaios que compuseram esta revisão não apresentaram um consenso acerca do tempo de reprodução e número de sessões necessárias para desencadear implicações na pressão arterial. No entanto, dentre aqueles que apresentaram tais informações, a maioria reproduziu a música de 20 a 30 minutos (80\%) e forneceu apenas uma sessão da intervenção (85\%).

Os achados ainda revelam que na grande maioria dos estudos (85\%), a música foi ofertada de forma receptiva e individualizada. Este resultado vai ao encontro dos achados de uma revisão realizada em 2014 sobre a mesma temática, em que $77,8 \%$ dos estudos utilizaram a forma receptiva da música, não sendo exposto se tais audições ocorreram de forma individual ou coletiva ${ }^{13}$.
De maneira similar, assim como em análise anterior $\frac{13}{3}$, os resultados aqui encontrados revelam a predominância do estilo musical clássico (35\%). Tal predomínio pode ser justificado pelas possíveis ações do estilo no organismo, proporcionando uma desaceleração e um comportamento mais ordenado; em contrapartida, melodias rápidas e repetitivas tendem a levar à agitação e euforia ${ }^{31}$.

Os resultados desta revisão permitem inferir que a música apresenta efeito positivo na redução da pressão arterial, mais especificamente na PAS, sendo tais implicações detectadas em pacientes adultos, em tratamento do câncer e em procedimentos cirúrgicos.

No estudo realizado em um ambulatório de pacientes com câncer de cabeça e pescoço, a música foi utilizada no período pós-cirúrgico imediato, após a quimioterapia e radioterapia, sendo que a análise indicou que $95 \%$ dos participantes do grupo experimental apresentaram redução da PAS e mais da metade tiveram redução da $\mathrm{PAD}{ }^{12}$.

Já um ensaio com pessoas que receberam raquianestesia, os resultados apontam que o grupo que recebeu a intervenção musical apresentou menor PAS e PAD, quando comparado ao grupo controle ${ }^{18}$. De modo semelhante, uma pesquisa que envolveu indivíduos com tumores cerebrais submetidos à craniotomia demonstrou que aqueles que escutaram música obtiveram menores médias e PAS e PAD $\stackrel{20}{ }$.

Um experimento realizado com pacientes em cirurgia de implante de lente intra-ocular indicou que a PAS foi significativamente menor no grupo exposto à música, enquanto que no grupo controle houve aumento desse parâmetro ${ }^{26}$.

Destaca-se que somente um estudo desta revisão foi direcionado à pacientes pediátricos. Neste, as crianças encontravam-se em ventilação mecânica e os resultados apontaram diminuição significativa da PAS após a intervenção da música, o que não foi observado na PAD $\underline{23}$.

O efeito da intervenção testada (música) sobre a variável de interesse (pressão arterial) pode ser atribuído à promoção do equilíbrio entre o sistema nervoso autônomo simpático e parassimpático. Nesse processo, é possível que a música tenha provocado uma diminuição da ação simpática e um aumento da atividade 
vagal, levando à diminuição do débito cardíaco e da resistência periférica e consequente restabelecimento dos níveis normais de pressão arterial. Acrescido a isso, pode-se inferir uma provável diminuição de catecolaminas (adrenalina e noradrenalina), seguida de vasodilatação e diminuiç̧ão dos valores pressóricos ${ }^{32}$.

Cabe destacar que os pesquisadores responsáveis pela execução dos ensaios aqui reunidos eram, em sua maioria, enfermeiros. Nessa vertente, tem-se que a música vem sendo utilizada por enfermeiros, ainda que de maneira empírica, desde o nascimento da enfermagem moderna. Florence Nightingale, grande precursora da profissão, utilizava tal estratégia para amenizar a dor de combatentes na Guerra da Criméia e promover o relaxamento. Ademais, os enfermeiros são os profissionais que acompanham diretamente o percurso do processo de adoecimento, o que pode levar a uma maior inquietação e anseio pelo desenvolvimento de pesquisas que visam a análise de terapias não usuais de assistência ${ }^{31}$.

Pode-se dizer que os achados desta revisão revelam o impacto da música no controle da pressão arterial, contudo, é importante destacar certas limitações. Primeiramente, a maioria dos ensaios realizou a mensuração da pressão arterial antes e após a intervenção, não identificando os possíveis efeitos a longo prazo. Outra limitação se refere a avaliação da pressão arterial, visto que grande parte dos estudos não apresentou o equipamento utilizado e a forma de avaliação do parâmetro fisiológico, o que pode tornar a interpretação dos dados mensurados pouco fidedignos. De modo semelhante, os ensaios não seguiram um protocolo de aplicação da música para avaliação de seus efeitos na pressão arterial, o que impossibilita a padronização, dificulta a reprodutibilidade de pesquisas e, consequentemente, a certificação de seus efeitos.

\section{Conclusão}

Os achados indicam efeito da música na pressão arterial, mais especificamente na diminuição da PAS. Identificou-se, que a maioria dos estudos que utilizam a música como terapia de controle da pressão arterial foram em pacientes adultos, em tratamento do câncer ou em procedimentos cirúrgicos. Isto posto, a intervenção com a utilização de música, por ser uma tecnologia leve, de baixo custo e de fácil aplicação, pode ser considerada um valioso recurso para o controle da pressão arterial.

Contudo, torna-se necessária a realização de ensaios que verifiquem os efeitos da intervenção a longo prazo e que sigam protocolos de avaliação da pressão arterial e de aplicação da música. Assim, será possível legitimar seus efeitos em diferentes populações e contextos e assegurar sua utilização visando o controle da pressão arterial.

\section{Contribuições dos autores}

Pereira JF participou da concepção e planejamento do projeto de pesquisa, obtenção, análise e interpretação dos dados e redação final. Souza MA e Assis FA participaram da análise e interpretação dos dados, redação final e revisão crítica. Souza PA e Luna AA participaram da redação final e revisão crítica. Silva NCM coordenou a pesquisa e participou da concepção e planejamento do projeto de pesquisa, análise e interpretação dos dados, redação final e revisão crítica.

\section{Conflitos de interesses}

Nenhum conflito financeiro, legal ou político envolvendo terceiros (governo, empresas e fundações privadas, etc.) foi declarado para nenhum aspecto do trabalho submetido (incluindo, mas não se limitando a subvenções e financiamentos, participação em conselho consultivo, desenho de estudo, preparação de manuscrito, análise estatística, etc.).

\section{Referências}

1. Organização Mundial da Saúde (OMS). Non communicable diseases [Internet]. 2018 [citado em 2019 out. 07]. Disponível em: https://www.who.int/en/news-room/fact-sheets/detail/ noncommunicable-diseases

2. Malta DC, Bernal RTI, Lima MG, Araújo SSC, Silva MMA, Freitas MIF et al. Doenças crônicas não transmissíveis e a utilização de serviços de saúde: análise da Pesquisa Nacional de Saúde no Brasil. Rev Saúde Pública. 2017;51(supl. 1). http://dx.doi. org/10.1590/s1518-8787.2017051000090

3. Hall JE, Guyton AC. Tratado de fisiologia médica. 13a.ed. Rio de Janeiro: Elsevier; 2017.

4. Sanjuliani AF. Fisiopatologia da hipertensão arterial: conceitos teóricos úteis para a prática clínica. Rev SOCERJ. [Internet]. 2002;15(4):210-218. Disponível em: http://sociedades.cardiol.br/ socerj/revista/2002_04/a2002_v15_n04_art02.pdf 
5. Sociedade Brasileira de Cardiologia. VII Diretriz Brasileira de Hipertensão Arterial. Revista da Sociedade Brasileira de Cardiologia. [Internet]. 2016;107 (3 supl 3). Disponível em: http:// publicacoes.cardiol.br/2014/diretrizes/2016/05_HIPERTENSAO_ ARTERIAL.pdf

6. Ministério da Saúde. Hipertensão é diagnosticada em $24,7 \%$ da população, segundo a pesquisa Vigitel. [Internet]. 2019. [citado em 2019 out. 07]. Disponível em: http://www.saude.gov.br/ noticias/agencia-saude/45446-no-brasil-388-pessoas-morrempor-dia-por-hipertensao

7. Ministério da Saúde. Hipertensão afeta um a cada quatro adultos no Brasil. [Internet]. 2019 [Citado em 2019 out. 07]. Disponível em: http://www.saude.gov.br/noticias/agenciasaude/45394-hipertensao-afeta-um-a-cada-quatro-adultos-nobrasil

8. Ministério da Saúde. Secretaria de Atenção à Saúde. Cadernos de Atenção Básica. Estratégias para o cuidado da pessoa com doença crônica-Hipertensão Arterial Sistêmica. Brasília: Ministério da Saúde; 2010.

9. Ministério da Saúde. Secretaria de Atenção à Saúde. Glossário temático: práticas integrativas e complementares em saúde. Brasília: Ministério da Saúde; 2018.

10. Ministério da Saúde. Portaria $n^{\circ} 971$, de 3 de maio de 2006. Aprova a Política Nacional de Práticas Integrativas e Complementares (PNPIC) no Sistema Único de Saúde. Diário Oficial da União. 2006 mai. 03.

11. Ministério da Saúde. Secretaria de atenção a saúde. Política nacional de práticas integrativas e complementares no SUS. 2a.ed. Brasília: Ministério da Saúde; 2018.

12. Firmeza MA, Rodrigues AB, Melo GAA, Aguiar MIF, Cunha $\mathrm{GH}$, Oliveira PP et al. Control of anxiety through music in a head and neckoutpatient clinic: a randomized clinical trial. Rev Esc Enferm USP. 2017;51:e03201. http://dx.doi.org/10.1590/S1980220X2016030503201

13. Santana DST, Zanini CRO, Sousa ALL. Efeitos da música e da musicoterapia na pressão arterial: uma revisão de literatura. In Cantare. [Internet]. 2014;5(1):37-57. Disponível em: http:// periodicos.unespar.edu.br/index.php/incantare/article/view/261

14. Gomes L, Amaral JB. Os efeitos da utilização da música para os idosos: revisão sistemática. Rev Enferm Contemp. 2012;1(1):103117. http://dx.doi.org/10.17267/2317-3378rec.v1i1.46

15. Moher D, Liberati A, Tetzlaff J, Altman DG. Preferred reporting items for systematic reviews and meta-analyses: the PRISMA statement. PLoS Med. 2009;6(7):1-6. http://dx.doi.org/10.1371/ journal.pmed.1000097
16. Jadad AR, Moore RA, Carroll D, Jenkinson C, Reynolds DJ, Gavaghan DJ et al. Assessing the quality of reports of randomized clinical trials: is blinding necessary? Control Clin Trials. 1996;17(1):1-12. http://dx.doi.org/10.1016/0197-2456(95)00134-4

17. Melo GAA, Rodrigues AB, Grangeiro ASM, Oliveira PP, Caetano JA. Musical intervention on anxiety and vital parameters of chronic renal patients: a randomized clinical trial. Rev Latino-Am Enfermagem. 2018;26:e2978. http://dx.doi.org/10.1590/1518$\underline{8345.2123 .2978}$

18. Kahloul M, Mhamdi S, Nakhi MS, Steyhi AN, Azzaza M, Chaouch $A$ et al. Effects of music therapy under general anesthesia in patients undergoing abdominal surgery. Libyan Journal Of Medicine. 2016;12:1,1260886. http://dx.doi.org/10.1080/1993282 $\underline{0.2017 .1260886}$

19. Lin CD, Marinova M, Rubino G, Gola E, Brocca A, Pantano $G$. Thoughts modulate the expression of inflammatory genes and may improve the coronary blood flow in patients after a myocardial infarction. Complement Ther Med. 2018;8(1):150-163. http://dx.doi.org/10.1016/j.jtcme.2017.04.011

20. Lee WP, Wu PY, Lee MY, Ho LH, Shih WM. Music listening alleviates anxiety and physiological responses in patients receiving spinal anesthesia. Complement Ther Med. 2017;31:8-13. http://dx.doi.org/10.1016/j.ctim.2016.12.006

21. Ripley L, Christopoulos G, Michael TT, Alomar M, Rangan $\mathrm{BV}$, Roesle $\mathrm{M}$ et al. Randomized controlled trial on the impact of music therapy during cardiac catheterization on reactive hyperemia index and patient satisfaction: the Functional Change in Endothelium After Cardiac Catheterization, With and Without Music Therapy (FEAT) study. J Invasive Cardiol. 2014;26(9):437-42.

22. Ko SY, Leung DYP, Wong EM. Effects of easy listening music intervention on satisfaction, anxiety, and pain in patients undergoing colonoscopy: a pilot randomized controlled trial. Clin Interv Aging. 2019;14:977-986. http://dx.doi.org/10.2147/CIA. $\underline{\text { S207191 }}$

23. Liu MH, Zhu LH, Peng JX, Zhang XP, Xiao ZH, Liu QJ et al. Effect of Personalized Music Intervention in Mechanically Ventilated Children in PICU: A Pilot Study. Pediatric Critical Care Medicine. 2019;21(1):1. http://dx.doi.org/10.1097/PCC.0000000000002159

24. Trappe HJ, Voit G. The cardiovascular effect of musical genres: a randomized controlled study on the effect of compositions by W. A. Mozart, J. Strauss, and ABBA. Dtsch Arztebl Int. 2016;113:347-52. http://dx.doi.org/10.3238/arztebl.2016.0347

25. Wu PY, Huang ML, Lee WP, Wang C, Shih WM. Effects of music listening on anxiety and physiological responses in patients undergoing awake craniotomy. Complement Ther Med. 2017;32:56-60. http://dx.doi.org/10.1016/j.ctim.2017.03.007 
26. Wiwatwongwana D, Vichitvejpaisal P, Thaikruea L, Klaphajone J, Tantong A, Wiwatwongwana A. The effect of music with and without binaural beat audio on operative anxiety in patients undergoing cataract surgery: a randomized controlled trial. Eye. 2016;30(11):1407-1414. http://dx.doi.org/10.1038/eye.2016.160

27. Ortega A, Gauna F, Munoz D, Oberreuter G, Breinbauer HA, Carrasco L. Music Therapy for Pain and Anxiety Management in Nasal Bone Fracture Reduction: Randomized Controlled Clinical Trial. Otolaryngol Head Neck Surg. 2019;161(4):613-619. http:// dx.doi.org/10.1177/0194599819856604

28. Choi S, Park SG, Bellan L, Lee HH, Chung SK. Crossover clinical trial of pain relief in cataract surgery. International Ophthalmology. 2017;38(3):1027-103. http://dx.doi.org/10.1007/ s10792-017-0554-y
29. Kavaklı AS, Ozturk NK, Adas HY, Kudsioglu ST, Ayoglu RU, Ozmen $S$ et al. The effects of music on anxiety and pain in patients during carotid endarterectomy under regional anesthesia: A randomized controlled trial. Complement Ther Med. 2019;44:94101. http://dx.doi.org/10.1016/j.ctim.2019.04.005

30. Azevedo E, Pelicioni MCF. Práticas integrativas e complementares de desafios para a educação. Trab Educ Saúde. 2011;9(3):361-378. http://dx.doi.org/10.1590/S1981$\underline{77462011000300002}$

31. Gonçalez DFC, Nogueira ATO, Puggina ACG. O uso da música na assistência de enfermagem no Brasil: uma revisão bibliográfica. Cogitare Enfermagem. 2008;13(4):591-596. http:// dx.doi.org/10.5380/ce.v13i4.13121

32. Nobre DV, Leite HR, Orsini M, Corrêa CL. Respostas Fisiológicas ao Estímulo Musical: Revisão de Literatura. Rev Neurocienc. 2012;20(4):625-633. http://dx.doi.org/10.34024/rnc.2012.v20.8232 